\title{
Como redigir um artigo original
}

\section{How to write an original article}

Izabel Cristina Meister Martins Coelho' (D) $\mid$ izabel.coelho@fpp.edu.br Pedro Tadao Hamamoto Filho² (1) pedro.hamamoto@unesp.br

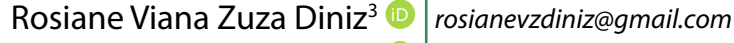
Suely Grosseman ${ }^{1,4}$ (D) sgrosseman@gmail.com

Visando contribuir para a compreensão dos aspectos centrais na elaboração de artigos e das particularidades que guiam a decisão do corpo editorial na análise de um manuscrito, a Revista Brasileira de Educação Médica (RBEM) inicia a série de editoriais para auxiliar os autores na construção dos manuscritos. Este texto é o primeiro dessa série que abordará as diversas modalidades de artigos científicos. Em primeiro lugar o texto apresenta as regras gerais e, em seguida, indica o detalhamento sobre cada seção de um artigo. $O$ objetivo é permitir que o leitor compreenda as especificidades para a redação de artigos originais.

\section{REGRAS GERAIS PARA ELABORAÇÃO DE PESQUISAS}

A possibilidade de publicação futura do estudo, quando envolve seres humanos, depende da submissão do projeto de pesquisa ao Comitê de Ética em Pesquisa (CEP) e à Comissão Nacional de Ética em Pesquisa (Conep). Somente após a aprovação, os dados (termo geralmente usado em pesquisas quantitativas) ou as informações (termo mais usado em pesquisas qualitativas) deverão ser coletados. Os pesquisadores precisam conhecer normas que regulam a ética da pesquisa. Para tanto, é imprescindível que leiam a Resolução no 466/2012', mais voltada a pesquisas biomédicas, a Norma Operacional no 001/2013² para o CEP/Conep e a Resolução no 510/2016 , voltada a pesquisas em Ciências Humanas e Sociais, frequentes no ensino na saúde. Apenas algumas situações justificam a não submissão do projeto de pesquisa ao CEP, explicitadas na Resolução n 510/20163:

Art. 1 [...] Parágrafo único. Não serão registradas nem avaliadas pelo sistema CEP/CONEP:

I- pesquisa de opinião pública com participantes não identificados;

II - pesquisa que utilize informações de acesso público, nos termos da Lei n 12.527, de 18 de novembro de 2011;

III - pesquisa que utilize informações de domínio público;

IV-pesquisa censitária;

$V$ - pesquisa com bancos de dados, cujas informações são agregadas, sem possibilidade de identificação individual; e

VI - pesquisa realizada exclusivamente com textos científicos para revisão da literatura científica;

VII - pesquisa que objetiva o aprofundamento teórico de situações que emergem espontânea e contingencialmente na prática profissional, desde que não revelem dados que possam identificar o sujeito; $e$

VIII - atividade realizada com o intuito exclusivamente de educação, ensino ou treinamento sem finalidade de pesquisa científica, de alunos de graduação, de curso técnico, ou de profissionais em especialização.

Vale ressaltar que, no contexto da educação médica e do ensino na saúde, a maioria das pesquisas precisa ser submetida ao comitê, como citado nos parágrafos seguintes da mesma resolução

$\S 1^{\circ}$ Não se enquadram no inciso antecedente os Trabalhos de Conclusão de Curso, monografias e similares, devendo-se, nestes casos, apresentar o protocolo de pesquisa ao sistema CEP/CONEP.

$\S 2^{\circ}$ Caso, durante o planejamento ou a execução da atividade de educação, ensino ou treinamento surja a intenção de

\footnotetext{
${ }^{1}$ Faculdades Pequeno Príncipe, Curitiba, Paraná, Brasil.

2 Universidade Estadual Paulista, Botucatu, São Paulo, Brasil.

3 Universidade Federal do Rio Grande do Norte, Natal, Rio Grande do Norte, Brasil.

${ }^{4}$ Universidade Federal de Santa Catarina, Florianópolis, Santa Catarina, Brasil.
}

Recebido em 05/03/21; Aceito em 05/03/21. 
incorporação dos resultados dessas atividades em um projeto de pesquisa, dever-se-á, de forma obrigatória, apresentar o protocolo de pesquisa ao sistema CEP/CONEP.

Projetos de pesquisa que envolvem uso de animais devem ser submetidos ao Comitê de Ética em Uso de Animais (Ceua). Já os ensaios randomizados devem seguir orientações do Consolidated Standards of Reporting Trials (CONSORT) 4 .

Para a submissão, o número da aprovação no Comitê de Ética ou do registro do protocolo de pesquisa nas bases apropriadas deve ser inserido na seção "Métodos", no corpo do manuscrito.

Outra regra fundamental é relativa ao plágio. Não se admite cópia de textos de outros autores ou de suas partes no manuscrito, sem colocá-la em forma de citação e referenciá-la. Isso se caracteriza como plágio, uma falha grave. Atualmente, mesmo a cópia de textos que o próprio autor do artigo publicou é considerado autoplágio.

\section{A PRODUÇÃO DO MANUSCRITO}

É importante selecionar o periódico ao qual submeterá o manuscrito antes de iniciar a redação. Alguns pontos a considerar para essa escolha são: aqueles que mais publicam artigos relacionados ao tema (escopo); o que teria interesse em publicar seus achados em função de sua abrangência (local, regional, nacional ou internacional); e o fator de impacto.

A elaboração do manuscrito obedece a instrução aos autores, pois cada periódico define o limite de palavras do artigo e a formatação de sua estrutura e das referências.

Uma boa revisão da literatura nacional e internacional deve ser robusta, de modo a fundamentar o referencial teórico para o estudo, pois trabalhar na fronteira do conhecimento de um tema requer sua abrangência mundial. É importante que as referências selecionadas apresentem concepções teóricas contemporâneas e sejam em sua maioria atuais, demonstrando o conhecimento dos autores no campo de pesquisa. Algumas referências antigas, que trazem autores que germinaram a construção do conhecimento e as perspectivas teóricas sobre o tema, denominadas clássicas, devem constar do referencial teórico. Como são frequentemente citadas nos artigos, essas referências são facilmente identificadas e são consideradas "raízes" ou estruturantes.

Outra recomendação sobre referências bibliográficas é evitar autocitação excessiva. $\mathrm{O}$ uso de autocitações que apenas tangenciam o tema é visto como estratégia de impulsionar indicadores bibliométricos do autor, o que caracteriza um comportamento científico antiético.

A utilização de softwares gratuitos de gerenciamento de referências bibliográficas, como o Mendeley ${ }^{5}$, Zotero ${ }^{6}$ e
EndNote ${ }^{7}$, auxilia o arquivamento e formatação das mesmas. Com o seu uso, as referências podem ser atualizadas de acordo com o formato exigido, caso seja necessária a submissão do artigo a outro periódico.

\section{A REDAÇÃO DO MANUSCRITO}

Nesta seção, cada componente da redação do artigo é detalhado considerando os critérios contidos em protocolos e diretrizes para a qualidade da elaboração, da redação e do impacto de pesquisa. Esses critérios são muito úteis e estão disponíveis no site Equator Network ${ }^{8}$, especialmente os contidos em The Strengthening the Reporting of Observational Studies in Epidemiology $(\mathrm{STROBE})^{9}$, Standards for reporting qualitative research (SRQR) ${ }^{10}$ e Consolidated criteria for reporting qualitative research (COREQ) ${ }^{11}$.

O título é a parte mais lida do artigo, e as buscas de artigos começam por sua leitura. Ele deve conter uma mensagem central que desperte o interesse do leitor e facilite sua compreensão sobre o objetivo do estudo, ser criativo, atraente e o mais curto possível (idealmente não ultrapassando 12 palavras), e conter um termo frequentemente usado no resumo.

O resumo deve seguir a estrutura requerida nas normas da revista. Habitualmente, ele abrange: contexto do estudo (introdução ou background) para situar o leitor; objetivo(s), tal como escrito(s) na introdução; método com detalhes suficientes sobre cada um de seus subitens; resultados com detalhes numéricos em pesquisas quantitativas e categorias e subcategorias, idealmente com alguns depoimentos marcantes em pesquisas qualitativas; e conclusões ou considerações finais.

Os editores de periódicos analisam detalhadamente o resumo, o qual é decisivo para a rejeição imediata do manuscrito ou seu encaminhamento aos revisores. Ainda, a qualidade do resumo de um artigo publicado atrai o leitor para acessar o artigo na íntegra ${ }^{12}$.

A introdução, como a própria palavra expressa, aproxima o leitor do tema. Deve ser breve e redigida para explicitar "aonde você quer chegar", "por que o seu estudo é necessário" e "para que/quem ele poderá ser útil". É composta pelos seguintes itens: contextualização do estudo, descrição do problema ou fenômeno abordado, definição de conceitos relevantes, conhecimentos já construídos sobre o tema, seus desfechos, relações causais e concepções teóricas/perspectivas existentes sobre o fenômeno de estudo. É estruturada por revisão abrangente e atualizada da literatura. Pontos fortes e limitações do conhecimento existente devem ser apontados para justificar a realização do estudo. Na pesquisa qualitativa, é importante articular as concepções teóricas/perspectivas que compuseram o seu referencial teórico e guiaram o seu olhar para abordagem do tema, gerando seus pressupostos. 
Finaliza-se a introdução com a(s) pergunta(s) de pesquisa e o(s) objetivo(s) do estudo, que devem ser claros e diretamente alinhados ao problema, e sua(s) hipóteses (pesquisas quantitativas) ou seus pressupostos (pesquisas qualitativas). Nos objetivos, evite adjetivos, use verbos de ação e explicite a população e o local da pesquisa ${ }^{13}$. Verbos comumente usados em pesquisas quantitativas são mais relacionados à mensuração e confirmação ou rejeição das hipóteses, como "analisar", "avaliar", "estimar" e "comparar"14, enquanto, em pesquisas qualitativas, estes são mais relacionados à compreensão do fenômeno, como "conhecer" e "compreender".

O método é a descrição do caminho percorrido para alcançar os objetivos. Ele permite que um leitor, ao apreciar o estudo, reproduza estudo similar. Portanto, a descrição deve ser abrangente e detalhada.

Inicia-se com a abordagem metodológica, o delineamento do estudo (tipo de estudo e se é descritivo ou analítico). Quando se adota a abordagem qualitativa, os motivos para essa opção devem ser explicados, inclusive para a escolha do referencial teórico e das estratégias para conhecer, compreender ou construir uma teoria sobre o fenômeno. Isso não só facilitará a compreensão dos leitores que não têm familiaridade com essa abordagem, como também esclarecerá por que o caminho escolhido foi considerado o mais adequado.

A seguir, devem ser descritos o local e os cenários em que o estudo foi realizado, indicando também as datas de realização do convite para participação no estudo, da coleta de dados/informações, da intervenção (se pertinente) e do acompanhamento dos desfechos. Se o estudo incluiu uma intervenção, esta deve ser descrita detalhadamente (local, duração, delineamento e avaliação).

Após, é imprescindível que sejam fornecidas informações claras e detalhadas sobre os participantes. Isso inclui o número de pessoas elegíveis, os critérios para inclusão e exclusão, o número estimado de participantes do estudo, o método de sua seleção (se aleatória ou se sistemática, explica-se como foi realizada; se intencional, explica-se a intenção dessa escolha; e, se por conveniência, explica-se como ela se deu, por exemplo, se foi pela presença das pessoas no dia da coleta de dados ou em horários específicos), local e forma de abordagem dos elegíveis para convidá-los a participar do estudo. Quando o estudo for controlado, os mesmos detalhes devem ser fornecidos para o grupo controle e para o pareamento, caso este seja realizado.

O número de participantes nas pesquisas qualitativas (diferentemente do requerido nas pesquisas quantitativas, que baseiam o tamanho da amostra em cálculos estatísticos) geralmente não ultrapassa o total de 25 a 30 pessoas, por visar ao aprofundamento da compreensão do fenômeno.

A coleta de dados ou de informações deve conter "o que, como, por quem e onde" foi realizada. Em pesquisa quantitativa, os dados são as variáveis estudadas, que incluem atributos gerais (como variáveis sociodemográficas), desfechos e seus fatores causais, bem como fatores com potencial de causar confusão na medida destes desfechos. Em pesquisas qualitativas, as informações coletadas geralmente incluem depoimentos, imagens e/ou reflexões e observações do pesquisador. É importante descrever de que fonte os dados foram coletados (por exemplo, de prontuários e protocolos) e os instrumentos, questionários, escalas para mensurar certas variáveis, fornecendo informações sobre a validade dos mesmos. Quando houver entrevistas abertas ou semiestruturadas, descreva quais foram as questões norteadoras. Caso o estudo seja de validação de um instrumento, devem ser fornecidos detalhes sobre como foi realizado o processo de validação e com base em que recomendações da literatura. As técnicas empregadas para a coleta dos dados/informações (como por exemplo questionário autoaplicado, observação participante ou não, entrevista, com detalhes sobre sua estruturação, grupo focal, entre outras), a duração média prevista, bem como sua forma de registro e tecnologias incluídas nesse processo (por exemplo, se escrito ou gravado com um dado equipamento em vídeo ou áudio e se houve ou não posterior transcrição) também são aspectos relevantes do método. Além disso, deve ser descrito o local em que os dados/as informações foram coletados. Na existência de registros escritos ou transcritos de depoimentos ou imagens, deve-se reportar se as informações foram retornadas (devolutiva) ou não para os participantes darem feedback. O projeto-piloto, que é parte importante de muitos estudos, também deve ser descrito, incluindo todos os detalhes acima.

Independentemente da abordagem metodológica, os aspectos do processo de coleta de dados/informações, especialmente quando há mensurações, entrevistas e grupos focais, devem ser referidas as características do "treinamento" prévio para a coleta e da avaliação do preparo. Neste aspecto, em pesquisas quantitativas são descritas as medidas de concordância e confiabilidade intra e interpesquisadores que coletaram os dados. Ainda, sugere-se descrever a experiência prévia e expertise dos pesquisadores ou coletores de dados/ informações para realizá-los, as relações estabelecidas com os participantes antes do início do estudo, o conhecimento prévio do participante sobre o entrevistador e as características do entrevistador (gênero e pressupostos), visto que todas essas podem inserir um viés no estudo.

$\mathrm{Na}$ análise de dados/informações, deve-se iniciar descrevendo os softwares usados e como foi analisada a qualidade do banco de dados. Em pesquisas quantitativas, apresentam-se os métodos estatísticos utilizados para cada 
tipo de análise e o nível de significância admitido para rejeitar a hipótese nula. Em pesquisas qualitativas, informa-se o tipo de análise usada, explicando o que foi feito em cada etapa dessa análise e aqui também devem ser descritos o tipo de treinamento prévio e a experiência das pessoas que fizeram a codificação (alguns autores preconizam inclusive que seja mencionado o sexo), como se chegou a um consenso sobre categorias e temas, e se houve feedback dos participantes também nessa etapa do estudo.

Os preceitos éticos, especificados nas regras gerais, devem constar no método.

Os resultados são iniciados com a descrição dos participantes (número, porcentagem do total e características). Em pesquisa quantitativa, é fornecido o detalhamento dos achados, junto com os resultados dos testes estatísticos. A frequência absoluta (número) e a frequência relativa (porcentagem) devem ser descritas. Detalha-se o número de participantes que não foram incluídos e as causas para a não inclusão (não compleição dos dados, por exemplo). Caso tenha havido perda de participantes ao longo do estudo (comum em estudos de coorte), devem ser explicados os procedimentos para lidar com esta perda. Fluxogramas para ilustrar a seleção, inclusão, exclusão e composição final da amostra são bemvindos. Resultados que possam ser descritos de forma breve não requerem tabelas ou figuras (por exemplo, número de participantes do sexo feminino e masculino), as quais devem ser usadas apenas quando a quantidade de dados a descrever tornaria a escrita textual muito longa e cansativa.

Em pesquisas qualitativas, é interessante apresentar-se inicialmente umafigura mostrandoascategorias e subcategorias encontradas. Depois, descreve-se cada tema, as categorias e subcategorias, com depoimentos ilustrativos, se necessários. Quando houver muitos depoimentos importantes, podese construir uma tabela com as categorias, as subcategorias e os depoimentos ilustrativos. Alguns autores preconizam identificar cada participante com uma letra e um número que não propiciem identificação, mas outros recomendam que isso não seja feito. Em nossa opinião, a identificação só é necessária quando há grupos diferentes, para propiciar a compreensão de diferenças e similaridades entre eles.

A discussão deve trazer uma síntese inicial dos principais achados, sem repetir o que já estava nos resultados, sua comparação com similaridades e diferenças de achados da literatura ou das perspectivas existentes, além da interpretação, considerações e reflexões dos autores sobre suas hipóteses e seus pressupostos iniciais e as justificativas para os achados de seu estudo ${ }^{15}$. Não deve haver apenas a citação dos resultados de trabalhos anteriores, sendo imprescindível promover um "diálogo" entre os achados do estudo, o de outros autores e as ideias dos autores. Além disso, é fundamental guiar-se pela pergunta "O que estou escrevendo na discussão está realmente relacionado aos resultados que encontrei ou poderia ter sido escrito antes mesmo de a pesquisa ser feita?". Se a resposta for "Não, essa parte poderia ter sido escrita antes mesmo da realização da pesquisa, pois não depende dos resultados encontrados", isso significa que essa parte não é pertinente à discussão. Portanto, deve-se ficar atento para não haver repetição do que já está explicitado na introdução. Um ponto crucial da discussão é a apresentação das limitações do estudo e do modo como elas podem ser superadas em investigações futuras. Não se pode esquecer-se de mencionar os próximos passos para melhor compreensão do tema por meio de novos estudos. Os pontos fortes do estudo, a aplicabilidade e a possibilidade de generalização em casos de pesquisa quantitativa finalizam a discussão.

As conclusões ou considerações finais devem responder às questões iniciais da pesquisa e aos seus objetivos, evitando dados ou ideias que não tenham sido discutidos no manuscrito.

Para encerrar, as referências bibliográficas devem estar formatadas conforme normas da revista.

Finalizando, espera-se que as sugestões apresentadas neste editorial, que podem ser aprofundadas em diversas fontes na literatura, contribuam para qualificar a publicação científica em educação médica no Brasil. Indicá-las neste editorial representa uma das ações do corpo editorial desta revista no sentido de orientar os autores de artigos originais, bem como guiar revisores de periódicos na elaboração de seus pareceres. Outras modalidades de artigos serão abordadas em editoriais futuros da RBEM.

\section{CONTRIBUIÇÃO DOS AUTORES}

Todos os autores colaboraram igualmente na confecção do artigo.

\section{CONFLITO DE INTERESSES}

Os autores declaram não haver conflito de interesses neste estudo.

\section{FINANCIAMENTO}

Declaramos que não houve financiamento para a realização desta pesquisa.

\section{REFERÊNCIAS}

1. Brasil. Resolução no 466, de 12 de dezembro de 2012. Trata sobre as diretrizes e normas regulamentadoras de pesquisa envolvendo seres humanos. Diário Oficial da União, Brasília, DF; 13 jun. 2013 [acesso em 22 fev 2021]. Disponível em: https://conselho.saude.gov.br/resolucoes/2012/ Reso466.pdf. 
2. Brasil. Norma Operacional $n^{\circ} 001$, de 12 de setembro de 2013. Dispõe sobre a organização e funcionamento do Sistema CEP/CONEP, e sobre os procedimentos para submissão, avaliação e acompanhamento da pesquisa e de desenvolvimento envolvendo seres humanos no Brasil. Diário Oficial da União, Brasília, DF; set 2013 [acesso em 18 fev 2021]. Disponível em: http://www.hgb.rj.saude.gov.br/ceap/Norma_Operacional_001-2013.pdf.

3. Brasil. Resolução $n^{\circ} 510$, de 7 de abril de 2016. Trata sobre as diretrizes e normas regulamentadoras de pesquisa em ciências humanas e sociais. Diário Oficial da União, Brasília, DF; 24 maio 2016 [acesso em 22 fev 2021]. Disponível em: http://bvsms.saude.gov.br/bvs/saudelegis/cns/2016/ res0510_07_04_2016.html.

4. CONSORT: transparent reports of trials [acesso em $22 \mathrm{fev} 2021$ ]. Disponível em: http://www.consort-statement.org/about-consort.

5. Mendeley Reference Manager. Elsevier, RLX Group [acesso em 20 fev 2021]. Disponível em https://www.mendeley.com/referencemanagement/reference-manager.

6. Zotero. Corporation for Digital Scholarship [acesso em 20 fev 2021]. Disponível em https://www.zotero.org/.

7. EndNote. Clarivate [acesso em 20 fev 2021]. Disponível em https:// endnote.com/.

8. Equator Network: Enhancing the QUAlity and Transparency of Health Research. University of Oxford [acesso em 27 fev 2021]. Disponível em https://www.equator-network.org/.
9. The Strengthening the Reporting of Observational Studies in Epidemiology (STROBE) statement: guidelines for reporting observational studies [acesso em 27 fev 2021]. Disponível em https://www.equatornetwork.org/reporting-guidelines/strobe/.

10. Standards for reporting qualitative research: a synthesis of recommendations [acesso em 27 fev 2021]. Disponível em https://www. equator-network.org/reporting-guidelines/srqr/.

11. Tong A, Sainsbury P, Craig J. Consolidated criteria for reporting qualitative research (COREQ): a 32-item checklist for interviews and focus groups. Int J Qual Health Care. 2007;19(6):349-57.

12. Valmi DS. Como escrever o resumo de um artigo para publicação. Acta Paul Enferm. 2006;19(3):5-8. doi: 10.1590/\$0103-21002006000300001.

13. Creswell JW. Projeto de pesquisa: método qualitativo, quantitativo e misto. 3a ed. Porto Alegre: Artmed, 2010.

14. Ferraz APCM, Belhot RV. Taxonomia de Bloom: revisão teórica e apresentação das adequações do instrumento para definição de objetivos instrucionais. Gestão \& Produção. 2010;17(2):421-31. doi: 10.1590/S0104$530 \times 2010000200015$

15. Campbell, C. Detailed guidelines for reporting quantitative research in Health \& Social Care in the Community. Health and Social Care in the Community, 2010. doi: 10.1111/j.1365-2524.2010.00962.x. 